\title{
Microfluidic Device for Enzyme-Linked Immunosorbent Assay (ELISA) and Its Application to Bisphenol A Sensing
}

\author{
Izumi Kubo*, Tomoyuki Kanamatsu and Shunsuke Furutani ${ }^{1}$ \\ Graduate School of Engineering, Soka University, 1-236 Tangi, Hachioji, Tokyo 192-8577, Japan \\ ${ }^{1}$ Health Research Institute, National Institute of Advanced Industrial Science and Technology, \\ 1-8-31 Midorigaoka, Ikeda, Osaka 563-8577, Japan
}

(Received January 15, 2014; accepted June 11, 2014)

Key words: $\quad$ bisphenol A, microfluidic disk, antibody-immobilized beads, competitive assay, rat serum

Bisphenol A (BPA) is a commonly used material made of polycarbonate, which is used in food containers. BPA is known to be an endocrine disruptor. To investigate the effect of BPA on health, animal experiments are necessary. To determine the intake of BPA in food or drink by animals, a BPA-sensing system that can detect a small amount of BPA in biological samples within a short reaction time is needed. We have developed a disc-shaped microfluidic device for enzyme linked immunosorbent assay (ELISA) with 32 microchannels and chambers. In order to establish a rapid and sensitive assay system for BPA in biological samples, in this study, anti-BPA antibody was immobilized on microbeads and introduced into microchambers on the microchannels in the device. A competitive immunoassay was performed using horse radish peroxidase (HRP)-conjugated BPA with a small amount of sample solution within 20 min. After the immune reaction, the HRP activity in each microchamber was detected by chemiluminescence. BPA could be determined on the microfluidic disk at a concentration range between 3.9 and 250 $\mathrm{ng} / \mathrm{ml}$. BPA-spiked rat serum was also determined on the disk.

\section{Introduction}

Some artificial chemicals are considered as environmental endocrine disruptors. Bisphenol A (BPA) is one such chemical. BPA is a major constituent of polycarbonate, which is widely used in food containers and feeding bottles because of its endurance to heat, durability and solidity. When hot food or drink is placed in a polycarbonate container, BPA present in the plastic tends to dissolve into the food or drink. The BPA

${ }^{*}$ Corresponding author: e-mail: kubo@soka.ac.jp 
eluted from the containers might be consumed with food and accumulate in the human body. There are fears related to the contamination from BPA in food or drink and its broad range of adverse effects on public health. ${ }^{(1)}$ In Japan, the concentration of BPA in food or drink must be lower than 2.5 ppm according to the Food Hygiene Law. Recently, however, it has been suspected that much lower concentrations of BPA may have adverse effects on life. For example, BPA perturbed the response of developmental programming of uterine estrogen ${ }^{(2)}$ or neuronal development at an early developmental stage ${ }^{(3,4)}$ in animal experiments. Such an effect on human health can be investigated by animal experiments. BPA concentration is conventionally measured by high-performance liquid chromatography (HPLC) or gas chromatography/mass spectrometry (GC/MS). The apparatus required for these methods is expensive, and the methods require sample pretreatment. However, from small animals such as mice, the amount of sample is often insufficient. Thus, a rapid and easy method for monitoring and analyzing BPA from a small amount of sample is required.

Microfluidics allows a rapid assay reaction and can accommodate a small volume of sample, making its application to enzyme linked immunosorbent assay (ELISA) pertinent and the focus of this study. A beads-based microfluidic ELISA chip ${ }^{(5-7)}$ and a microfluidic device utilizing centrifugal force for liquid flow ${ }^{(8,9)}$ have been reported. These systems effectively reduce assay time, although high throughput screening is not easily performed owing to the difficulty of parallel reactions of many reaction chambers. These devices were originally developed for the detection of disease markers. Microfluidic ELISA for the detection of BPA has not been reported. In this study, we established a BPA sensing system using our original custom-made microfluidic ELISA disklike device, which utilizes centrifugal force to introduce liquid into reaction chambers, enabling us to perform parallel reactions in many chambers. We apply this system to determine BPA concentration. Detection of BPA was performed through a competitive immunoassay mixed with horse radish peroxidase (HRP)-conjugated BPA. The system was applied to determine BPA in rat serum.

\section{Materials and Methods}

\subsection{Materials}

Rabbit anti-BPA antibody (rabbit anti-Bisphenol A-CME-BSA IgG; Cosmobio, Japan) and HRP-conjugated BPA solution (Bisphenol A-4-CPE-HRP; Cosmobio, Japan) were the commercialized reagents used for ELISA of BPA. For the blocking reaction after the immobilization of antibody, Block Ace (DS Pharma, Japan) and Starting Block $^{\mathrm{TM}}$ (PBS) (Thermo Fisher Scientific) were used. The chemiluminescent HRP substrate was picoluminol (Thermo Fisher Scientific). As buffer solution for ELISA, 0.1 M phosphate buffer (pH 7.0) and Tris buffered saline and Tween20 (TBST) (20 mM Tris$\mathrm{HCl}, 0.15 \mathrm{M} \mathrm{NaCl}, 0.05 \%$ Tween 20 , pH 7.6) were used.

As photoresist used for microfluidic patterning, SU-8 2035, SU-8 2005, and SU-8 developer were purchased from MicroChem. Polydimethylsiloxane (PDMS; Sylgard 184 silicone elastomer kit) was purchased from World Precision Instruments, Inc. 


\subsection{Design and fabrication of microfluidic disk}

Microfluidic disks were designed and prepared according to a previous study ${ }^{(10)}$ although the designs of microchannels and chambers were slightly modified. The design of the microfluidic disk $(\phi=10 \mathrm{~cm})$ is shown in Fig. 1(a). Thirty-two Y-shaped radial channels were organized on one disk. One channel consisted of a reaction chamber, an outlet and two inlets, one for the reagents of the immunoreaction and the other for the enzyme substrate, as shown in Fig. 1(b). The channel width was $100 \mu \mathrm{m}$, and its depth was large $(60 \mu \mathrm{m})$ from the inlets to the end of the microchamber and shallow $(15 \mu \mathrm{m})$ at the end of the chamber to keep the antibody-immobilized beads in the chamber. An elliptical reaction chamber $\left(1000 \times 500 \mu \mathrm{m}^{2}\right)$ was arranged on each channel. To keep the introduced liquid in the reaction chamber, it was followed by the turns of microchannel to the inlet and to the vent again [Fig. 1(b)]. This part is different from the previously reported microfluidic disk design.

The microfluidic disk was fabricated by soft photolithography, as follows. A negative photoresist (SU-8 2005) was spun onto a silicon wafer using a spin coater (model K-359 S-1, Kyowa Riken, Tokyo, Japan) at 3000 rpm for 30 s. Photolithography using a photomask patterned with microchannels was performed according to previously reported procedures. $^{(11)}$ To fabricate deep microchannels (depth $\left.=60 \mu \mathrm{m}\right)$, SU-8 2035 was subsequently coated on a SU-8 2005 layer at $3000 \mathrm{rpm}$ for $30 \mathrm{~s}$ and exposed to UV light through the photomask prior to development. Using the template patterned with SU-8, microchannels were molded out of 1-mm-thick PDMS. The PDMS microchannel disk was covered with a glass plate (thickness $=0.7 \mathrm{~mm}$ ). Inlets were made by perforation of the PDMS disk.

\subsection{Preparation of antibody-immobilized beads}

Rabbit anti-BPA antibody (BPA Ab) was immobilized on the surface of carboxylated beads (polybeads carboxylated $\phi 20 \mu \mathrm{m}$; Cosmobio, Japan) by the carbodiimide method as follows. A suspension of beads was maintained for $1 \mathrm{~h}$ in water-soluble carbodiimide solution ( $1 \mathrm{mg} / \mathrm{ml}, \mathrm{pH} 5.0)$. After spinning and rinsing in the phosphate buffer $(0.1 \mathrm{M}$, $\mathrm{pH}$ 7.0), beads were suspended in BPA Ab solution and kept at $4{ }^{\circ} \mathrm{C}$, overnight. After

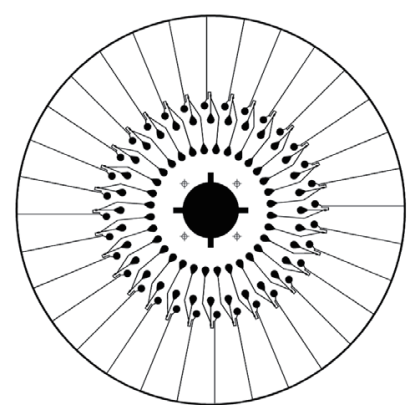

(a)

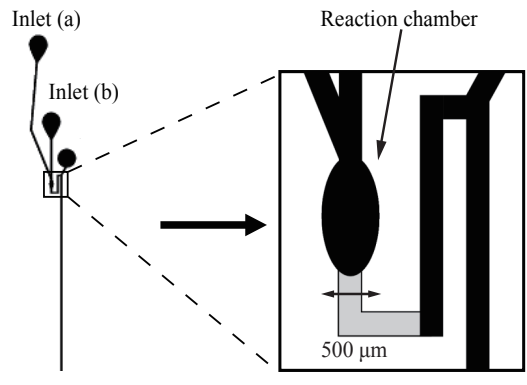

(b)

Fig. 1. Microfluidic disk for ELISA. (a) Microfluidic disk. (b) One channel with a reaction chamber. 
blocking with Block Ace or Starting Block ${ }^{\mathrm{TM}}, 2.0 \times 10^{3}$ particles of the Ab-immobilized beads were injected into the inlet and introduced into the reaction chamber by rotation (5000 $\mathrm{rpm}, 30 \mathrm{~s})$.

\subsection{Immunoassay}

Competitive ELISA of BPA using a microtiter plate with 96 wells was performed as follows. Rabbit anti-BPA antibody (BPA Ab) was immobilized to the plate (Nunc 442404). For the competitive assay, equal volumes of sample solution containing BPA and HRP-conjugated BPA were mixed and used in the assay. Fifty microliters of the mixture of sample solution and HRP-conjugated BPA, which was diluted to $10^{4}$-fold of the source reagent, was placed in each well and reacted with the antibody for $3 \mathrm{~h}$ at room temperature. Assays were performed in triplicate. After the reaction, HRP activity was measured using luminol as substrate, and luminol chemiluminescence (CL) of each well was determined.

ELISA steps using the disk were as follows (Fig. 2). First, the mixture $(1 \mu \mathrm{l})$ of equal volumes of sample solution containing BPA and HRP-conjugated BPA solution was injected into inlet (a). The centrifugal disk was rotated to transport the sample solution into the reaction chamber to bind to the Ab-immobilized microbeads. Every rotation was performed at $5000 \mathrm{rpm}$ for $30 \mathrm{~s}$. The injection of $1 \mu \mathrm{l}$ of the mixture and the rotation of the disk was repeated three times for the complete introduction of the mixture into the reaction chamber. The disk was incubated at room temperature for $15 \mathrm{~min}$ to perform an antigen-antibody reaction. Next, TBST buffer solutions were injected into inlet (a), and the centrifugal disk was rotated to wash the reaction chamber. This washing procedure was repeated five times to remove unreacted HRP-conjugated BPA. Finally, $1 \mu 1$ of HRP substrate solution was injected into another inlet (b) to avoid the background CL caused by trace amounts of HRP-conjugated BPA remaining in inlet (a). The centrifugal disk was rotated to transport the HRP substrate solution into the reaction chamber. Immediately after the reaction, CL was measured with an imaging analyzer (LAS-3000, FUJIFILM). Each assay was performed in triplicate. All sample concentrations were simultaneously determined in this procedure. ELISA was completed within 20 min with this procedure, and multiple experiments, as many as 32 assays, were simultaneously performed with the same disk.

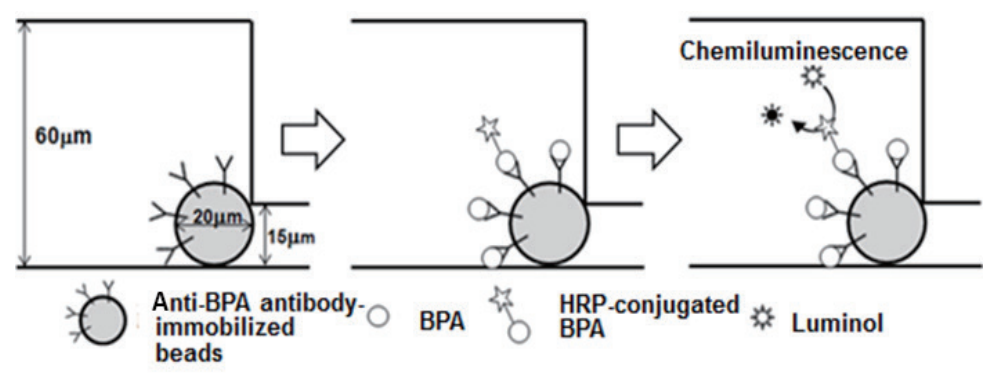

Fig. 2. Scheme of ELISA of BPA on a microfluidic disk. 


\subsection{Pretreatment of rat serum}

A solid phase extraction (SPE) column (Isolute SPE M-M; Biotage, Japan) was used for the pretreatment of rat serum spiked with BPA according to the SPE method, recommended by Biotage. For the conditioning, $10 \mathrm{ml}$ of methanol and ultrapure water were passed through the column, and $100 \mu \mathrm{l}$ of rat serum containing BPA was applied. After the application, the column was washed with $6 \mathrm{ml}$ of $35 \%$ methanol, and bound BPA was eluted with $2.5 \mathrm{ml}$ of methanol. The eluent was completely dried by bubbling nitrogen gas, and dissolved in 10\% methanol. The extracted BPA in 10\% methanol was used in ELISA.

\section{Results and Discussion}

\subsection{BPA competitive assay using conventional ELISA}

Prior to the immobilization of the antibody on microbeads, competitive ELISA using a microtiter plate was carried out. As a result, a calibration plot for BPA was obtained in which BPA was determined at a concentration range between 0.97 and $1000 \mathrm{ng} / \mathrm{mL}$ (Fig. 3). To carry out the microtiter plate assay, $210 \mathrm{~min}$ in total and $80 \mu \mathrm{l}$ of sample were required for a triplicate assay.

\subsection{BPA ELISA on microfluidic device}

To optimize the immobilization of BPA $\mathrm{Ab}$, the concentration of the $\mathrm{Ab}$ was examined at $2.0 \times 10^{4}$-, $1.0 \times 10^{4}$ - and $5.0 \times 10^{3}$-fold dilutions. Ab of $1.0 \times 10^{4}$-fold dilution showed the largest signal after the binding of HRP-conjugated BPA.

To examine the blocking effect of microchannels, chambers and beads, Block Ace, PBS and no blocking agent were compared. Prior to the introduction of Ab-immobilized beads, microchannels and chambers were filled with these solutions, left for $15 \mathrm{~min}$, then washed with TBST buffer. When Block Ace and Starting Block ${ }^{\mathrm{TM}}$ were used, competitive reactions were apparently observed (Fig. 4). Blocking with Block Ace resulted in a 3-fold higher CL signal than that with Starting Block ${ }^{\mathrm{TM}}$. Without blocking,
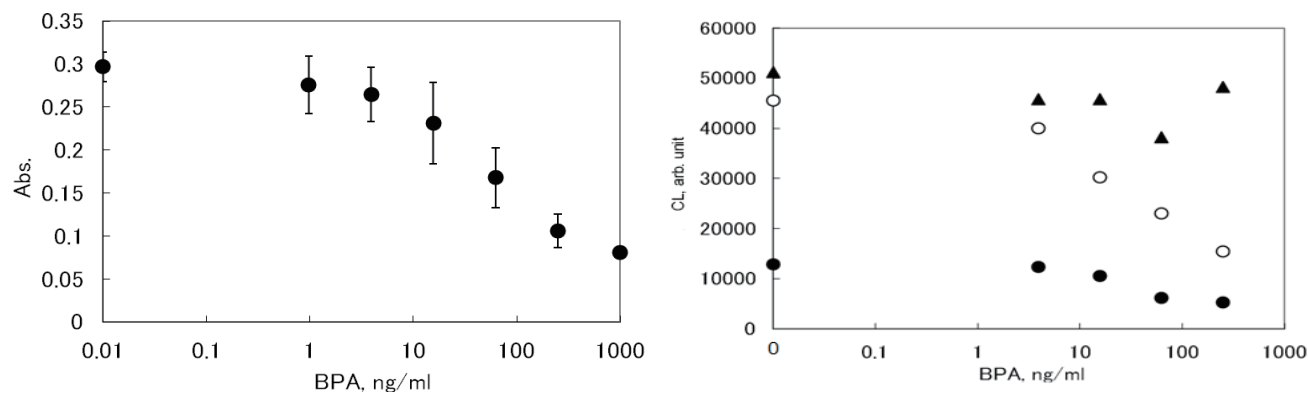

Fig. 3 (left). Calibration plot of BPA competitive ELISA on a microtiter plate. Experiment was performed in triplicate.

Fig. 4 (right). Effect of blocking reagent on ELISA. ○ Block ace, • Starting block, $\boldsymbol{\Delta}$ No blocking. 
no effective competition was observed. In this study, Block Ace was used to block the microfluidic disk and beads.

Then, BPA was determined using the microfluidic disk. In each reaction chamber, 2.0 $\times 10^{3} \mathrm{Ab}$-immobilized beads were introduced. Competitive immunoassay was performed by applying an equal volume mixture of HRP-conjugated BPA diluted $10^{4}$-fold and BPA solution to the determination chamber, followed by incubation for $15 \mathrm{~min}$. After washing the chamber with $5 \mu \mathrm{l}$ of buffer solution (TBST), CL substrate was introduced into the chamber and detected. As CL substrate, luminol and picoluminol were examined, and picoluminol showed a 50-fold higher CL signal than that shown by luminol. Therefore picoluminol was used to assay BPA in this study. The dilution rate of HRP-conjugated BPA was examined at $1.0 \times 10^{4}$-fold to $8.0 \times 10^{4}$-fold; $2.0 \times 10^{4}$-fold showed the largest CL signal. Under these conditions, BPA could be calibrated. CL signals depended on the concentration of BPA in the range of 3.9-250 ng/ml (Fig. 5). This shows that by utilizing Ab-immobilized beads on the microfluidic disk, with a small amount of sample solution, BPA could be determined in a much shorter reaction time than on a microtiter plate. To compare the calibration plot obtained using a microfluidic disk with that using the microtiter plate, each signal was normalized by the signal at $0 \mathrm{mg} / \mathrm{ml} \mathrm{BPA}$ (Fig. 6). Almost the same change in signals relative to the concentration of BPA was observed. This system can be applied to the determination of a small amount of sample.

\subsection{Determination of BPA in rat serum}

As a real serum sample, rat serum spiked with BPA was examined. From the spiked rat serum, BPA was extracted with an SPE column (Isolute SPE M-M). The extracted BPA was determined with the microfluidic disk. The spiked concentrations were 40 and $400 \mathrm{ng} / \mathrm{ml}$. From the calibration plot, the concentration of each sample was evaluated (Table 1).

An almost consistent CL signal was obtained, but at a higher concentration (400 ng/ $\mathrm{ml}$ ), the evaluated concentration was lower than the spiked value. A calibration plot of a higher concentration was not examined on the disk because of the limited number of
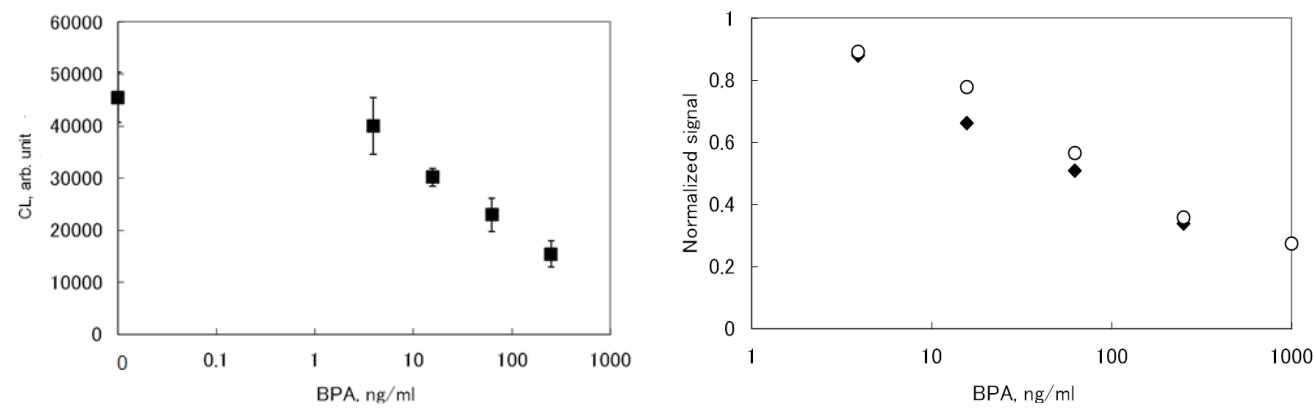

Fig. 5 (left). Calibration plot of BPA competitive ELISA on a microfluidic disk. Experiment was performed in triplicate.

Fig. 6 (right). Comparison of ELISA on a microfluidic disk and a microtiter plate. Microfluidic disk, o Microtiter plate. 
Table 1

BPA in rat serum determined by microfluidic disk ELISA.

\begin{tabular}{lc}
\hline Spiked conc. of BPA & Determined by microfluidic ELISA \\
\hline $40.0 \mathrm{ng} / \mathrm{ml}$ & $40.7 \pm 0.7 \mathrm{ng} / \mathrm{ml}$ \\
\hline $400 \mathrm{ng} / \mathrm{ml}$ & $320 \pm 68.2 \mathrm{ng} / \mathrm{ml}$ \\
\hline
\end{tabular}

microchambers. If a more precise determination is required, a calibration plot of a broad range should be examined.

In animal experiments, mice were treated with BPA of $5 \mathrm{mg} / \mathrm{kg}$, ${ }^{(2)}$ which corresponds to the $5 \mu \mathrm{g} / \mathrm{ml}$. Even when $1 \%$ of BPA $(50 \mathrm{ng} / \mathrm{ml})$ is in the circulation pool, it can be detected using the microfluidic disk.

\section{Conclusions}

With the use of the microfluidic disk filled with antibody-immobilized polybeads, the same competitive reaction as in a microtiter plate assay was performed. The volume of sample solution was $5 \mu \mathrm{l}$ for a triplicate assay, which is almost $1 / 15$, while the determination time was reduced to $1 / 10$ compared with that for the microtiter plate assay. BPA was determined on the microfluidic disk in a concentration range of between 3.9 and $250 \mathrm{ng} / \mathrm{ml}$ within $20 \mathrm{~min}$. This method will be very useful for assaying BPA from a limited amount of sample, such as in experiments involving small test subjects or animals.

\section{Acknowledgements}

This work was supported in part by a Grant-in-Aid for Scientific Research (C) from the Ministry of Education, Culture, Sports, Science and Technology (MEXT) of Japan and in part by a MEXT-Supported Program for the strategic Research Foundation at Private University, S1001013, 2010-2015.

\section{References}

1 Y. Ikezuki, O. Tsutsumi, Y. Takai, Y. Kamei and Y. Taketani: Hum. Reprod. 17 (2002) 2839.

J. G. Bromer, Y. Zhou, M. B. Tayler, L. Doberty and H. S. Taylor: FASEB J. 24 (2010) 2273.

K. Nakamura, K. Itoh, T. Sugimoto and S. Fushiki: Neurosci. Lett. 420 (2007) 100.

4 K. Kawai, S. Murakami, E. Senba, T. Yamanaka, Y. Fujiwara, S. Arimura T. Nozaki, M. Takii and C. Kubo: Regul. Toxicol. Pharmacol. 47 (2007) 166.

5 W. T. Liu, L. Zhu, Q. W. Qin, Q. Zhang, H. Feng and S. Ang : Lab Chip 5 (2005) 1327.

6 T. Ohashi, K. Mawatari, K. Sato, M. Tokeshi and T. Kitamori: Lab Chip 9 (2009) 991.

7 M. Herrmann, E. Roy, T. Veres and M. Tabrizian: Lab Chip 7 (2007) 1546.

8 S. Lai, S. Wang, J. Luo, L. J. Lee, S. T. Yang and M. J. Madou: Anal. Chem. 6 (2004) 1832.

9 H. Nagai, Y. Narita, M. Ohtaki, K. Saito and S. Wakida: Anal. Sci. 23 (2007) 975.

10 N. Matsunaga, S. Furutani and I. Kubo: ECS Transactions 16(11) (2008) 123.

11 S. Furutani, H. Nagai and I. Kubo: Sens. Lett. 6 (2008) 961. 